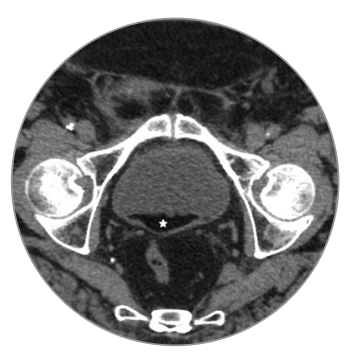

\title{
Nivel líquido-grasa intravesical secundario a perforación vesical silente tras resección transuretral de carcinoma urotelial
}

\author{
Intravessical Fat-Fluid Level Secondary to Silent Bladder Perforation \\ After Transurethral Resection of Urothelial Carcinoma
}



\section{Key words (MeSH)}

Urinary bladder

Carcinoma transitional cell

Ultrasonography

Multidetector computed

tomography

\footnotetext{
'Licenciada(o) especialista en Radiodiagnóstico. Servicio de Radiodiagnóstico. Hospital Santiago Apóstol. Miranda de Ebro, España.
}

\begin{abstract}
Resumen
Los tumores vesicales no músculo-invasivos se definen como lesiones papilares limitadas a la mucosa vesical o que invaden la lámina propia, además de los tumores de morfología plana y alto grado limitados a la mucosa (carcinoma in situ [CIS]). La confirmación diagnóstica se produce tras el análisis histológico de la muestra obtenida en la resección transuretral (RTU) de vejiga, que en los tumores Ta-T1 requiere la resección completa de todas las lesiones, incluyendo parte del músculo detrusor, lo que constituye, también, el método terapéutico. En el caso del CIS, que puede simular inflamación o no ser visible en la cistoscopia, son necesarias múltiples biopsias aleatorias de la vejiga. La perforación iatrogénica de la vejiga es el segundo evento adverso más frecuente del mencionado procedimiento; esta solución de continuidad en la pared puede dar lugar a la migración de grasa hacia el interior de la vejiga, que en las pruebas de imagen se traduce en la formación de un nivel líquido-grasa intravesical, hallazgo infrecuente, que a su vez posee un amplio diagnóstico diferencial, que abarca causas primarias, infecciosas y traumáticas, pero no previamente descrito como una complicación de la RTU. Se presenta el caso de un paciente asintomático con un nivel líquido-grasa intravesical secundario a perforación extraperitoneal de vejiga, tras la resección transuretral de un carcinoma urotelial no músculo-invasivo en la cúpula vesical.
\end{abstract}

\section{Summary}

Non-muscle-invasive bladder tumours are defined as papillary lesions limited to the bladder mucosa or invading the lamina propia, in addition to flat morphology and high-grade tumors limited to the mucosa (carcinoma in situ [CIS]). Diagnostic confirmation occurs after histological analysis of the sample obtained in the transurethral resection of the bladder (TURB), which in Ta-T1 tumours requires complete resection of all lesions, including part of the detrusor muscle, being in these cases also the therapeutic method. In the case of CIS, which may simulate inflammation or not be visible in cystoscopy, multiple random bladder biopsies are necessary (2). latrogenic bladder perforation is the second most frequent adverse event of this procedure; This solution of continuity in the wall can lead to the migration of fat into the bladder, which in imaging tests results in the formation of an intravesical fat-fluid level, a rare finding, which in turn has a broad differential diagnosis that covers primary, infectious and traumatic causes, but not previously described as a complication of TURB. We report the case of an asymptomatic patient with presence of intravesical fat-fluid, secondary to extra-peritoneal bladder perforation, after transurethral resection of a non-muscleinvasive urothelial carcinoma in the bladder dome.

\section{Abreviaturas \\ CIS (carcinoma in situ), RTU (resección transuretral), TC (tomografía computarizada)}

\section{Introducción}

Según la clasificación TNM (1), el carcinoma urotelial de vejiga no músculo-invasivo se define como un tumor papilar limitado a la mucosa vesical (Ta) o que invade la lámina propia (T1), además de los tumores de morfología plana y alto grado limitados a la mucosa (carcinoma in situ [CIS]). La confirmación diagnóstica se produce mediante el análisis histológico de la muestra obtenida en la resección transuretral (RTU) de vejiga — que en los tumores Ta-T1 requiere la resección completa de 
todas las lesiones, incluido parte del músculo detrusor - mientras que en el caso del CIS - que puede simular inflamación o no ser visible en la cistoscopia - se necesitan múltiples biopsias aleatorias de la vejiga (2). Entre las complicaciones de la RTU se destacan la hematuria $(2,8 \%)$ y la perforación vesical $(1,3 \%)$ (3). La mayoría de estas perforaciones son extraperitoneales $y$, cuando son sintomáticas, suelen resolver con sondeo vesical y profilaxis antibiótica; por el contrario, cuando la rotura se extiende hacia el espacio intraperitoneal requiere reparación quirúrgica (4). En casos excepcionales, una rotura vesical no diagnosticada puede persistir en el tiempo y ser detectada posteriormente - de forma incidental, en las pruebas de imagen - como en el paciente de este caso, a quien durante un estudio de imagen de control se le identificó líquido-grasa intravesical y una pequeña herniación de tejido graso a través de una solución de continuidad en la pared vesical

\section{Descripción del caso}

Hombre de 79 años que, como antecedentes de interés, presentaba tres episodios de RTU de vejiga: en 1999 por un carcinoma urotelial Ta, en 2007 por recidiva de carcinoma urotelial T1, y en 2018 por un CIS localizado en la cúpula vesical. Pocos días después de la última RTU ingresó por un cuadro de disuria, polaquiuria y fiebre, acompañado de malestar general y molestias en la región suprapúbica, el cual tuvo una evolución favorable con tratamiento antibiótico, se le dio el alta y ha permanecido asintomático desde entonces.

Seis meses después, en la primera ecografía de control, se detectó un artefacto de reverberación en el hipogastrio junto a la región anterior de la vejiga, que no permitía la valoración completa de las paredes de esta, inicialmente interpretado como gas intestinal.

Por esa razón, se realizó una urografía por TC (UroTAC) (figura 1), con el paciente en apnea y con técnica de Split bolus, que incluyó una adquisición del abdomen y pelvis sin medio de contraste y otra adquisición combinada (fases nefrográfica y excretora) tras la administración de medio de contraste intravenoso (inyección inicial de $60 \mathrm{~mL}$ a una velocidad de $3 \mathrm{~mL} / \mathrm{s}$, después de 10 minutos una inyección adicional de $70 \mathrm{~mL}$ a $3 \mathrm{~mL} / \mathrm{s}$ y, finalmente, obtención de las imágenes 65 segundos después). Mediante esta técnica se comprobó que el artefacto ecográfico era causado por grasa intravesical, que conformaba un nivel líquidograsa en la región anterior; además, se identificaron colecciones líquidas laminares extraperitoneales en el espacio perivesical y una laceración en la cúpula vesical, a través de la cual se observaba herniación de una pequeña cantidad de grasa perivesical.

A continuación, se realizó una cistografía por tomografía dinámica (figura 2), previa distensión retrógrada de la vejiga con medio de contraste yodado, que demostró extravasación a través del defecto de la pared y hacia el espacio perivesical del medio de contraste, acompañado de pequeñas burbujas de gas introducido durante la manipulación; dicha fuga era visible durante la maniobra de Valsalva y tras la micción, pero no en la serie inicial con la vejiga llena, que se obtuvo antes de alcanzar la máxima distensión.

Adicionalmente, se practicó una TC de pelvis en decúbito prono (figura 3), que evidenció cómo el componente graso intravesical adoptaba una posición no dependiente, al igual que lo observado en la UroTAC en decúbito supino. La cistoscopia confirmó la existencia de partículas de grasa suspendidas dentro de la vejiga, pero no permitió la visualización de la rotura vesical.

\section{Discusión}

La perforación vesical iatrogénica puede ser secundaria a un traumatismo externo (procedimientos obstétricos, ginecológicos, generales o urológicos) o interno, como es el caso de la RTU de vejiga (4). Tiene una incidencia documentada del 2-13\%; sin embargo, se ha descrito que su incidencia real puede llegar a 50-58 \%, según lo demuestran varios estudios en los que se realizó cistoscopia o cistografía de forma sistemática tras la RTU (5), pero la mayoría son asintomáticas. Estas perforaciones pueden ser intraperitoneales, con una extravasación de líquido hacia la cavidad peritoneal, alrededor de las asas intestinales, entre las capas mesentéricas y en las goteras paracólicas; o extraperitoneales, en las que la fuga se limita al espacio perivesical o se extiende a otros planos fasciales y espacios pélvicos (6).


Figura 1. UroTAC. a) Axial y b) sagital, en fases nefrográfica y excretora. Se observa grasa intravesical (estrella), colecciones líquidas laminares en el espacio perivesical (flecha blanca) y una laceración en la cúpula vesical (flecha roja), a través de la cual se hernia una pequeña cantidad de grasa perivesical. 

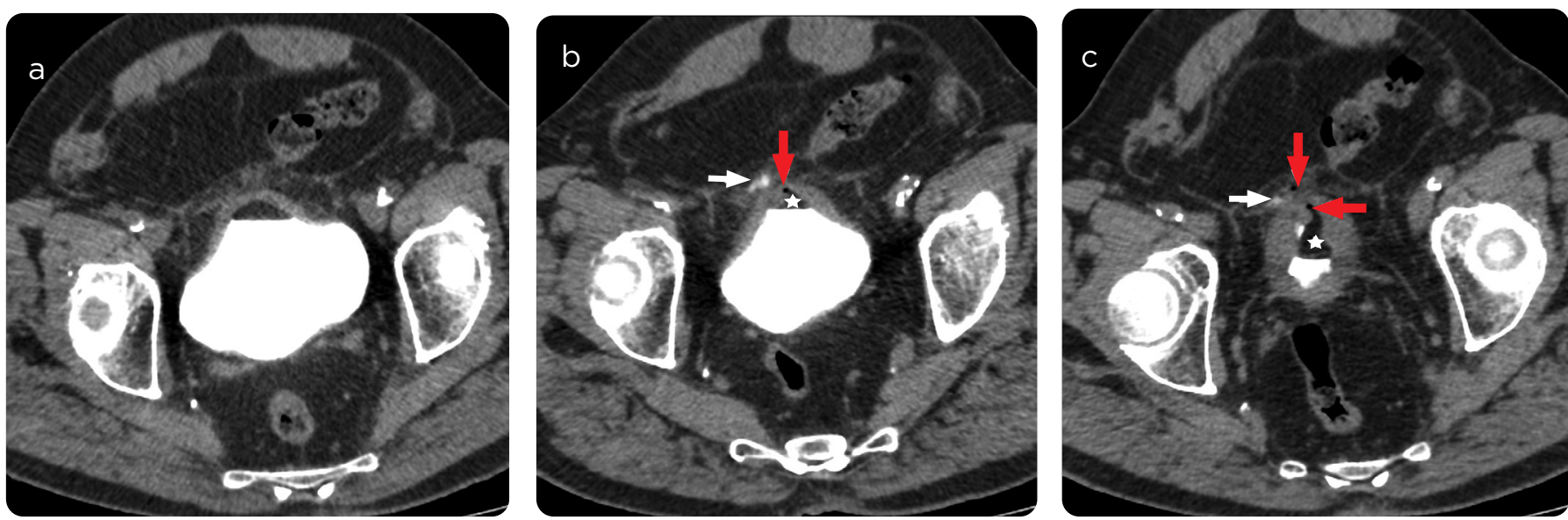

Figura 2. TC-cistografía en decúbito supino, a) axial con la vejiga llena, pero no a máxima distensión, sin evidencia de extravasación. b) Axiales durante la maniobra de Valsalva y c) tras la micción: se identifica extravasación al espacio perivesical de medio de contraste (flecha blanca) y de gas introducido durante la manipulación (flecha roja). Grasa intravesical (estrella) en posición antideclive.

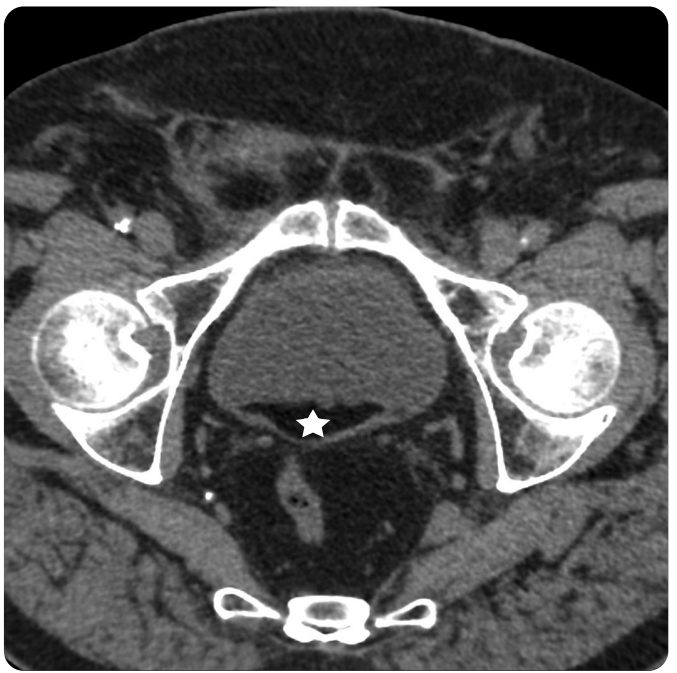

Figura 3. TC de pelvis en decúbito prono, axial: el componente graso intravesical (estrella) se desplaza con los movimientos del paciente hasta una posición antideclive.

Al contrario de las perforaciones vesicales grandes, que habitualmente se diagnostican en el acto quirúrgico, muchas lesiones menores pasan desapercibidas y solo se detectan en el postoperatorio, cuando dan lugar a manifestaciones clínicas (hematuria, dolor suprapúbico, disfunción de vaciado vesical, distensión abdominal, íleo, peritonitis o sepsis), mediante cistoscopia o pruebas de imagen (4). El $74 \%$ de las perforaciones extraperitoneales se resuelven con medidas conservadoras en 10-14 días, y el $85 \%$ en un mes, mientras que los casos asintomáticos o con una evolución subclínica, en los cuales no se produce la curación espontánea de la lesión debido a la herniación de grasa perivesical a su través (7), suelen tener un diagnóstico tardío, meses o años después.

Aunque la cistografía convencional ha sido la técnica de elección ante la sospecha de perforación vesical iatrogénica (4), la cistografía por TC con distensión vesical retrógrada tiene una sensibilidad diagnóstica de aproximadamente el $100 \%$, según pequeñas series de casos (6), además de una mayor precisión para definir el tipo de rotura, delimitar las zonas anatómicas afectadas y detectar lesiones en las estructuras adyacentes, como el tracto intestinal, gracias en parte a su capacidad multiplanar (8). Para evitar falsos negativos es imprescindible evaluar la vejiga bajo presión, con una distensión máxima (8); de lo contrario, se recomienda adquirir series adicionales durante la maniobra de Valsalva o tras el vaciado vesical (posmiccional o con sonda Foley).

La UroTAC con técnica de Split bolus se caracteriza porque divide en dos inyecciones el medio de contraste intravenoso y adquiere simultáneamente las fases nefrográfica y excretora. Tiene las ventajas de disminuir la dosis de radiación y permitir la valoración en una única serie del parénquima renal y del sistema excretor, así como del parénquima de los demás órganos abdominales y estructuras venosas; su principal limitación es que aumenta ligeramente la dosis de medio de contraste yodado que es necesario administrar. Entre sus indicaciones se encuentran la valoración de las litiasis del tracto urinario, las alteraciones renales y los tumores uroteliales (9).

La grasa intravesical, cuyos valores de atenuación en TC oscilan entre -80 y -100 unidades Hounsfield, se denomina lipiduria y da lugar a la formación de un nivel líquido-grasa, en el que el componente graso se desplaza con los movimientos del paciente y adopta siempre una posición no dependiente, como se observó en el estudio en decúbito supino y decúbito prono. Este infrecuente hallazgo debe distinguirse de un nivel líquido-gas intravesical mediante un adecuado ajuste de las ventanas de visualización y medición de las unidades Hounsfield, del depósito de grasa intramural — que es un hallazgo benigno y puede ser focal o difuso - y de un verdadero lipoma, que consiste en tejido graso encapsulado en la submucosa de la pared vesical.

Existen otras causas de grasa intravesical que pueden clasificarse en: 1) traumáticas, como en el caso de las fracturas pélvicas, 2) posquirúrgicas a nefrectomía parcial, ablación percutánea de tumores renales, litotricia extracorpórea, hemicolectomía, esofagectomía, entre otras, 3) médicas, como la filariosis linfática (por Wuchereria bancrofti, Echinococcus, cisticercosis, malaria y ascariasis), infecciones granulomatosas (TBC), abscesos, embarazo, adenitis mesentérica masiva, enfermedad glomerular, aneurisma aórtico, neoplasias y malformaciones del sistema linfático (10). 


\section{Conclusión}

El hallazgo de nivel líquido-grasa intravesical en TC tiene múltiples diagnósticos diferenciales, que abarcan desde causas primarias hasta causas traumáticas e iatrogénicas, sin que se encuentre descrito previamente en la literatura la RTU de carcinoma urotelial de vejiga como una de ellas, por lo que en este caso se demuestra que, ante el antecedente de dicho procedimiento, se debe valorar la opción de una perforación vesical iatrogénica.

\section{Referencias}

1. Brierley JD, Gospodarowicz MK, Wittekind C. TNM classification of malignant tumours. 8th ed. Oxford: Wiley-Blackwell; 2017.

2. Babjuk M, Böhle A, Burger M, Capoun O, Cohen D, Compérat EM, et al. EAU guidelines on non-muscle-invasive urothelial carcinoma of the bladder: Update 2016. Eur Urol. 2017;71:447-61.

3. Collado A, Cechile GE, Salvador J, Vicente J. Early complications of endoscopic treatment for superficial bladder tumors. J Urol. 2000;164:1529-32.

4. Summerton DJ, Kitrey ND, Lumen N, Serafetinidis E, Djakovic N. European Association of Urology. EAU guidelines on iatrogenic trauma. Eur Urol. 2012;62:628-39.

5. Balbay MD, Cimentepe E, Unsal A, Bayrak O, Koc A, Akbulut Z. The actual incidence of bladder perforation following transurethral bladder surgery. J Urol. 2005;174:2260-2.

6. Vaccaro JP, Brody JM. CT cystography in the evaluation of major bladder trauma. Radiographics. 2000;20:1373-81.

7. Lim AK, Johns Putra LG, Troy AJ, Ierino FL. Intravesical fat entrapment as a cause of failure of extraperitoneal bladder perforation to heal spontaneously. Int Urol Nephrol. 2007:39:795-8.

8. Chan DP, Abujudeh HH, Cushing GL Jr, Novelline RA. CT cystography with multiplanar reformation for suspected bladder rupture: experience in 234 cases. AJR Am J Roentgenol. 2006;187:1296-302.

9. Chow LC, Kwan SW, Olcott EW, Sommer G. Split-bolus MDCT Urography with synchronous nephrographic and excretory phase enhancement. AJR Am J Roentgenol. 2007;189:314-22.

10. Tan Y. Chyluria in non-filarial endemic areas: an internist's perspective. BMJ Case Reports. 2017;2017:bcr-2017-220772.

\section{Correspondencia}

Nancy Bibiana Aristizábal Buitrago

Servicio de Radiodiagnóstico, Hospital Santiago Apóstol

Carretera de Orón s/n. 34004

Miranda de Ebro, España.

nbaristizabal@saludcastillayleon.es

Recibido para evaluación: 18 de marzo de 2020

Aceptado para publicación: 26 de abril de 2020 